\title{
Piezoelectric anisotropy: Enhanced piezoelectric response along nonpolar directions in perovskite crystals
}

\author{
D. DAMJANOVIC, M. BUDIMIR, M. DAVIS, N. SETTER \\ Ceramics Laboratory, Materials Institute, Ecole Polytechnique Fédérale de Lausanne-EPFL, \\ 1015, Lausanne, Switzerland \\ E-mail:dragan.damjanovic@epfl.ch
}

This paper discusses the mechanisms that can contribute to the enhanced longitudinal piezoelectric effect along nonpolar directions in perovskite crystals, such as $\mathrm{BaTiO}_{3}, \mathrm{PbTiO}_{3}$, $\mathrm{KNbO}_{3}, \mathrm{~Pb}\left(\mathrm{Mg}_{1 / 3} \mathrm{Nb}_{2 / 3}\right) \mathrm{O}_{3}-\mathrm{PbTiO}_{3}$ and $\mathrm{Pb}\left(\mathrm{Zn}_{1 / 2} \mathrm{Nb}_{2 / 3}\right) \mathrm{O}_{3}-\mathrm{PbTiO}_{3}$. Piezoelectric anisotropy is discussed in relation to temperature induced phase transitions, compositional variation in solid solutions with morphotropic phase boundaries, applied electric fields, the domain wall structure and domain wall displacement. (c) 2006 Springer Science + Business Media, Inc.

\section{Introduction}

The anisotropy of elastic, dielectric and piezoelectric properties plays an important role in the application of piezoelectric materials. Several types of the anisotropy have been discussed in the literature, including the orientation dependence of the material coefficients and the difference in the values of the longitudinal, transverse and shear coefficients. The two best known examples are the temperature stabilized cuts of quartz [1] and $\mathrm{LiNbO}_{3}$ crystals [2] where the temperature dependences of different elastic compliances compensate, leading to low temperature coefficients of resonant frequencies and acoustic velocities. Anisotropy of piezoelectric properties was intensively studied in the 1980s when unusually strong piezoelectric anisotropy was found in lead titanate ceramics with random grain orientation [3]. In these materials, for example, the longitudinal $d_{33}$ coefficient behaves in the expected way whereas the transverse coefficient $d_{31}$, which in most perovskite materials is negative and 2-4 times smaller than $d_{33}$, can be close to zero or can even exhibit a positive sign [4]. Another example is the strong anisotropy of dielectric, elastic and piezoelectric properties in textured ceramics of bismuth based Aurvillius structures [5]. The anisotropy of the elastic and piezoelectric properties is exploited in piezoelectric-ceramic composites with 1-3 connectivity to reduce lateral coupling in transducers for underwater and medical imaging applications [6].
Interest in the electromechanical anisotropy of ferroelectric materials has been spectacularly renewed in the last several years after the (re)discovery [7, 8] of the large electromechanical coupling coefficient $k_{33}(>90 \%)$ and longitudinal piezoelectric coefficient $d_{33}(>2000 \mathrm{pC} / \mathrm{N})$ measured along nonpolar directions in relaxor-ferroelectric solid solutions [e.g., $\mathrm{Pb}\left(\mathrm{Mg}_{1 / 3} \mathrm{Nb}_{2 / 3}\right) \mathrm{O}_{3}-\mathrm{PbTiO}_{3}$ or PMN-PT and $\mathrm{Pb}\left(\mathrm{Zn}_{1 / 3} \mathrm{Nb}_{2 / 3}\right) \mathrm{O}_{3}-\mathrm{PbTiO}_{3}$ or PZN-PT]. The reason why this result was surprising can probably be found in the fact that the most widely used piezoelectric material over the last 50 years has been ceramic $\mathrm{Pb}(\mathrm{Zr}, \mathrm{Ti}) \mathrm{O}_{3}$. Poled ceramics exhibit conical symmetry and their largest longitudinal, transverse and shear piezoelectric responses are measured along the axes of the orthogonal coordinate system whose $x_{3}$ axis lies parallel to the poling (polar) direction [9]. A maximum of the longitudinal piezoelectric response along nonpolar directions, as observed in relaxorferroelectrics, was therefore unexpected. However, subsequent studies have found that such behavior is common and has been reported in many perovskite crystals, both in those having simple compositions (e.g., $\mathrm{BaTiO}_{3}[10]$ and $\mathrm{KNbO}_{3}[11]$ ) and in other complex solid solutions (e.g., $\mathrm{BiScO}_{3}-\mathrm{PbTiO}_{3}$ [12] and $\mathrm{Pb}\left(\mathrm{Yb}_{1 / 2} \mathrm{Nb}_{1 / 2}\right) \mathrm{O}_{3}-\mathrm{PbTiO}_{3}$ [13]). Similar anomalies have been observed for the transverse and shear coefficients. In contrast to poled ceramics, the transverse coefficient is found to be largest in a plane that is not perpendicular to the polar direction. The shear 
effect behaves oppositely and is the highest when the field is applied and response measured along the corresponding axes of the crystallographic coordinate system [14, 15]. As we shall see later these results are nontrivial as some important perovskites, like $\mathrm{PbTiO}_{3}$ [16], behave similarly to ceramics, exhibiting highest piezoelectric effects along the axes of the crystallographic coordinate system.

While the origin of the large piezoelectric activity in crystals of complex solid solutions (e.g., PMN_PT, PZNPT) is still not clear, the related research has led to unprecedented activity in the field of ferroelectric materials, directly or indirectly leading to the discovery of the monoclinic phase in $\mathrm{Pb}(\mathrm{Zr}, \mathrm{Ti}) \mathrm{O}_{3}$ [17], to the development of textured ceramics with enhanced piezoelectric properties

$$
a=\left(\begin{array}{c}
\cos \psi \cos \varphi-\cos \theta \sin \varphi \sin \psi \\
-\sin \psi \cos \varphi-\cos \theta \sin \varphi \cos \psi \\
\sin \theta \sin \varphi
\end{array}\right.
$$

[18], and to the renewed interest in the role of engineered domain states on the electromechanical properties of ferroelectric crystals. Interpretation of experimental results obtained on single crystals has been further complicated by the fact that virtually all experimental data have been reported for multidomain samples, and the role of the intrinsic anisotropy and the presence of the engineered domain structure have not been separated.

In this article we shall discuss selected aspects of the piezoelectric anisotropy in perovskite crystals, focusing on mechanisms that may contribute to the enhancement of the longitudinal $d_{33}$ piezoelectric coefficient when measured along nonpolar directions. The text is structured in the following way. First, basic relations defining the orientation dependence of piezoelectric coefficients in the tetragonal $4 m m$, orthorhombic $\mathrm{mm} 2$, and rhombohedral $3 m$ point groups are given and briefly discussed in Section 2. The piezoelectric anisotropy is then discussed in Section 3 in relation to the proximity of ferroelectricferroelectric phase transitions, in Section 4 in terms of composition in materials exhibiting a morphotropic phase boundary, in Section 5 as a function of external electric fields, and in Section 6 in terms of the domain wall structure. Finally the effects of the extrinsic contributions on the electromechanical response are briefly discussed in Section 7.

\section{Orientation dependence of the piezoelectric coefficients}

The piezoelectric coefficient is a tensor of the third rank that can be transformed between two coordinate systems using the relation [19]:

$$
d_{i j k}^{*}(\varphi, \theta, \psi)=a_{i l} a_{j m} a_{k n} d_{l m n}
$$

where $\varphi, \theta, \psi$ are the Euler angles and $a_{i j}$ are the elements of the Euler matrix that describes the rotation defined by the Euler angles. In this text $d$ is the tensor of piezoelectric coefficients in the crystallographic coordinate system with axis $x_{1}, x_{2}$ and $x_{3}$, while the asterisk denotes the tensor in the rotated system. A caution is necessary when comparing data from different sources since Euler angles are not uniquely defined in the literature. Here we follow the definition of Euler angles given in Refs. $[2,20]$ where $\varphi$ describes the first counterclockwise rotation around the $x_{3}$ axis, $\theta$ the second counterclockwise rotation around the new $x_{1}$ axis and $\psi$ the third counterclockwise rotation around the new $x_{3}$ axis. The corresponding rotation matrix is:

$$
\left.\begin{array}{cc}
\cos \psi \sin \varphi+\cos \theta \cos \varphi \sin \psi & \sin \psi \sin \theta \\
-\sin \psi \sin \varphi+\cos \theta \cos \varphi \cos \psi & \cos \psi \sin \theta \\
-\sin \theta \cos \varphi & \cos \theta
\end{array}\right)
$$

If another definition of the Euler angles is used, the terms in equations given below may have different signs $[21,22]$ and numerical coefficients. Furthermore, the piezoelectric tensor is usually given in the form of a matrix with reduced indices using Voigt notation [19]. A mistake sometimes made in the literature is to use for the piezoelectric $d$ coefficients the reduced matrix of the third rank electro-optical tensors or the piezoelectric stress tensor $e$ [23]. The reduced matrices for these tensors may differ from the matrix for the $d$ tensor in the relationship between the coefficients. For example, in point group $3 m$ in the reduced notation $d_{26}=-2 d_{11}$ but $e_{26}=-e_{11}$ [24].

In this article we shall discuss only the orientation dependence of the longitudinal $d_{33}$ piezoelectric coefficient. This coefficient has the simplest form and is the easiest to analyze, yet allows a point to be made about the main features of anisotropy that will be discussed. In the most common symmetries and for the chosen definition of the Euler angles, the Equation 1 has the following forms:

in crystals belonging to the tetragonal $4 \mathrm{~mm}$ group:

$$
d_{33}^{t *}(\theta)=\cos \theta\left(d_{15}^{t} \sin ^{2} \theta+d_{31}^{t} \sin ^{2} \theta+d_{33}^{t} \cos ^{2} \theta\right)
$$

in the orthorhombic $m m 2$ group:

$$
\begin{aligned}
d_{33}^{o *}(\theta, \phi)= & \cos \theta\left[\left(d_{15}^{o}+d_{31}^{o}\right) \sin ^{2} \theta \sin ^{2} \phi+\left(d_{24}^{o}\right.\right. \\
& \left.\left.\left.+d_{32}^{o}\right) \sin ^{2} \theta \cos ^{2} \phi+d_{33}^{o} \cos ^{2} \theta\right)\right]
\end{aligned}
$$

and in the rhombohedral $3 m$ group:

$$
\begin{aligned}
d_{33}^{r *}(\theta, \phi)= & d_{15}^{r} \cos \theta \sin ^{2} \theta-d_{22}^{r} \sin ^{3} \theta \cos 3 \phi \\
& +d_{31}^{r} \sin ^{2} \theta \cos \theta+d_{33}^{r} \cos ^{3} \theta
\end{aligned}
$$




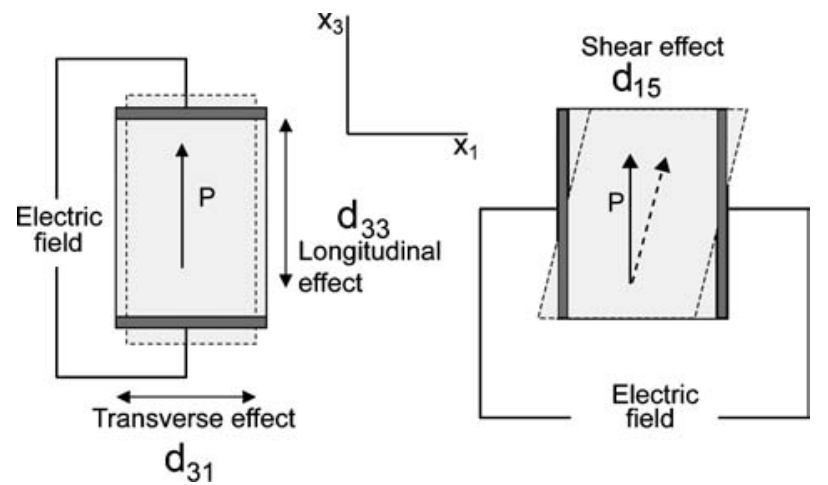

Figure 1 Illustration of longitudinal, transverse and shear piezoelectric effects in a tetragonal material and piezoelectric ceramic. The dark regions at the sample edges represent electrodes.

Indices $t, o$ and $r$ refer to the crystal symmetries. In the rest of the text terms 'tetragonal', 'orthorhombic' and 'rhombohedral' will refer to point groups $4 \mathrm{~mm}, \mathrm{~mm} 2$, and $3 m$. Definitions of the longitudinal, transverse and shear coefficients are given in Fig. 1.

\section{Piezoelectric anisotropy in the proximity of temperature induced ferroelectric-ferroelectric phase transitions}

Let us consider $\mathrm{BaTiO}_{3}$ mono domain single crystals. This material is of interest since it undergoes a series of phase transitions (cubic $\rightarrow$ tetragonal $\rightarrow$ orthorhombic $\rightarrow$ rhombohedral) as it is cooled from the paraelectric phase [25]. These crystal phases are typical of perovskite ferroelectrics and this fact, together with its chemical simplicity, makes $\mathrm{BaTiO}_{3}$ convenient for modeling purposes. The temperature dependences of piezoelectric coefficients of $\mathrm{BaTiO}_{3}$ are experimentally available only for the tetragonal phase [26], however, the coefficients of the Gibbs free energy expansion are known so that piezoelectric coefficients can be predicted as a function of temperature in all three phases, as shown in

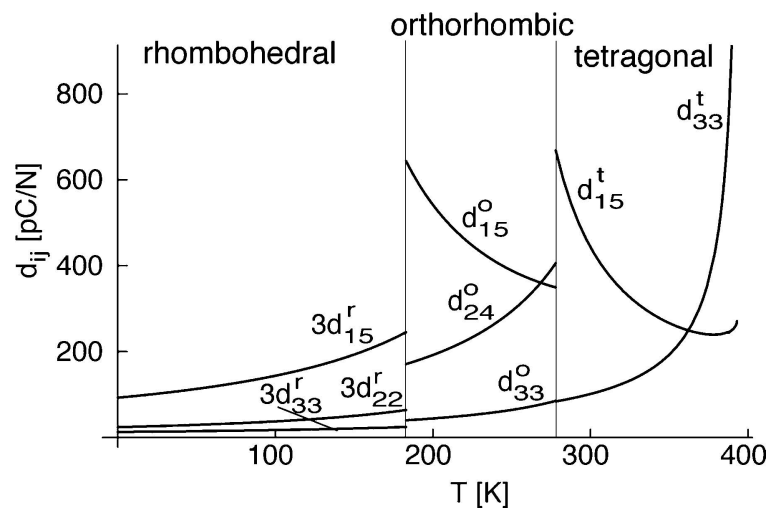

Figure 2 Temperature dependence of piezoelectric coefficients in all three ferroelectric phases of $\mathrm{BaTiO}_{3}$ predicted by the LGD theory. Parameters of the LGD function were taken from Ref. [27].

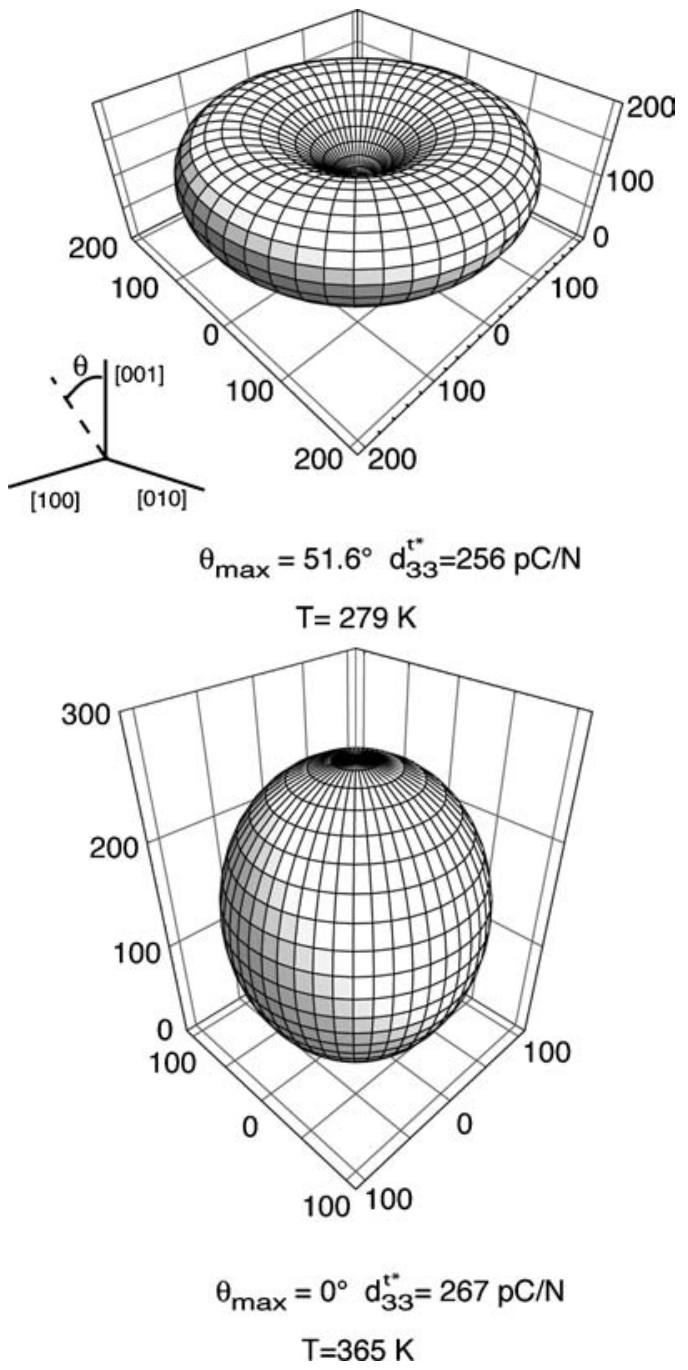

Figure 3 Orientation dependence of $d_{33}^{t *}(\theta)$ in the tetragonal phase of $\mathrm{BaTiO}_{3}$ at two temperatures, close to the tetragonal-orthorhombic (upper figure) and the tetragonal-cubic (lower figure) phase transition temperature. Numbers on axes indicate values of $d_{33}^{t *}(\theta)$. The coordinate system indicates crystallographic axes.

Fig. 2. The orientation dependence of piezoelectric coefficients, $d_{33}^{*}(\theta, \varphi)$, can be calculated at any temperature using Equations 3-5, as discussed in Ref. [16]. Behavior of $d_{33}^{*}(\theta, \varphi)$ in the tetragonal, Fig. 3, and the orthorhombic, Fig. 4, phases illustrates how orientation dependence changes with temperature and as the phase transition temperatures are approached.

The evolution of the $d_{33}^{t *}(\theta)$ surface near the tetragonalorthorhombic and the orthorhombic-rhombohedral phase transitions temperatures can be analyzed and understood on several levels. The simplest approach is by inspecting the Equations 3-4 and Fig. 2 and by analyzing the competing influences of the shear (term with $\sin \theta$ ) and the longitudinal (term with $\cos \theta$ ) piezoelectric coefficients, while neglecting for simplicity the influence of the small transverse coefficient $d_{31}^{t}$. In the tetragonal phase the $d_{33}^{t *}(\theta)$ 


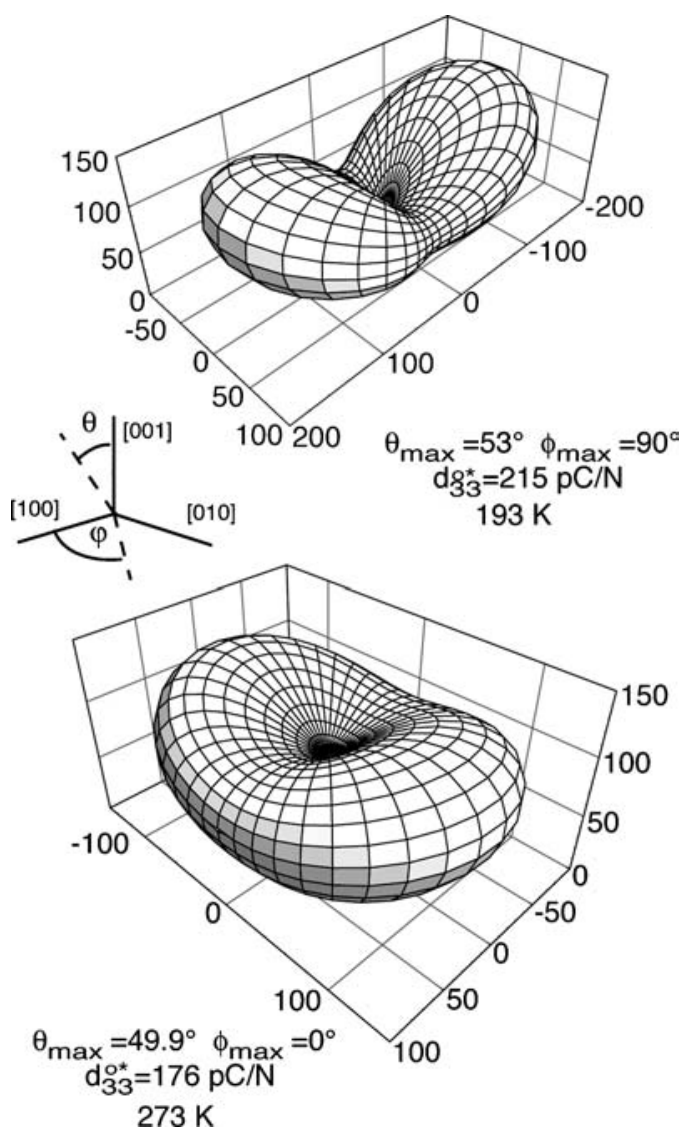

Figure 4 Orientation dependence of $d_{33}^{o *}(\theta)$ in the orthorhombic phase of $\mathrm{BaTiO}_{3}$ at two temperatures, close to the rhombohedral-orthorhombic (upper figure) and the orthorhombic-tetragonal (lower figure) phase transition temperature. Numbers on axes indicate values of $d_{33}^{o *}(\theta)$. The coordinate system indicates crystallographic axes.

exhibits maximum value along the polar axis as long as the ratio of the shear and longitudinal coefficients, $d_{15}^{t} / d_{33}^{t}$, is below some critical value [28]. When this is no longer the case (i.e., when $d_{15}^{t}$ is sufficiently high), $d_{33}^{t *}(\theta)$ will exhibit a maximum along a nonpolar direction. As can be expected from Fig. 3 the direction along which $d_{33}^{t *}(\theta)$ exhibits maximum is strongly temperature dependent [28].

More complex behavior is predicted for the orthorhombic phase, Fig. 4, which has two different shear coefficients, one dominating near the orthorhombicrhombohedral and the other near the orthorhombictetragonal phase transition temperature, Fig. 2. In this phase, as is the case for the rhombohedral one, the longitudinal piezoelectric coefficient is always largest along a nonpolar direction. The rotation of the $d_{33}^{o *}(\theta)$ surface with temperature is directly caused by the change of the shear coefficient that dominates expression (4). For details see Ref. [16].

In the rhombohedral phase of $\mathrm{BaTiO}_{3}$, the form of the $d_{33}^{r *}(\theta, \varphi)$ surface is typical of that found in other materials with rhombohedral $3 m$ symmetry, Fig. 5. The $d_{33}^{r *}(\theta, \varphi)$ surface has its maximum approximately along the [001]

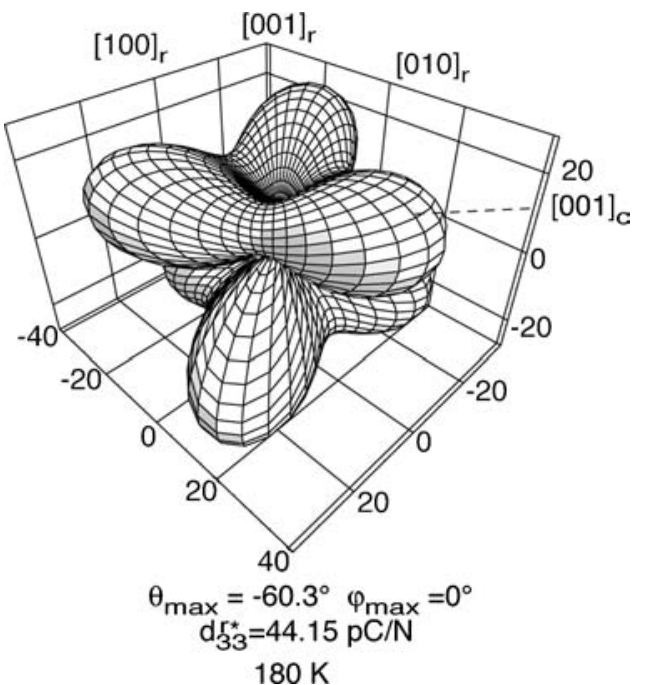

Figure 5 Orientation dependence of $d_{33}^{r *}(\theta)$ in the rhombohedral phase of $\mathrm{BaTiO}_{3}$ at $180 \mathrm{~K}$. The form of the surface does not change as the temperature is decreased. Numbers on axes indicate values of the $d_{33}^{r *}(\theta)$. The orientation of crystallographic axes is indicated by $[h k l]$. The maxiumum $d_{33}^{r *}(\theta)$ lies near the quasicubic $\langle 001\rangle_{c}$ direction.

pseudocubic direction, just as in complex relaxor ferroelectrics. The form of $d_{33}^{r *}(\theta, \varphi)$ remains constant with temperature, and the anisotropy (the ratio between the maximum $d_{33}^{r *}(\theta, \varphi)$ and $d_{33}^{r}$ along the polar direction) slightly decreases as the temperature is decreased [16].

It is useful at this point to comment on the very large piezoelectric response along off polar axes in relaxor ferroelectrics, such as PMN-PT and PZN-PT. The anisotropy in these materials is giant [14, 15, 23]. For example, the measurements of the properties of monodomain 0.67PMN-0.33 PT crystals show $d_{33}^{r}$ of about $190 \mathrm{pC} / \mathrm{N}$ and $d_{15}^{r}>4000 \mathrm{pC} / \mathrm{N}$ in the crystallographic coordinate system, leading to maximum $d_{33}^{r *}(\theta, \varphi)>2400 \mathrm{pC} / \mathrm{N}$ along a nonpolar direction $[15,22]$. Thus, in the simplest approach [in terms of Equation 3], it is the large $d_{15}^{r}$ which is responsible for the large $d_{33}^{r *}(\theta, \varphi)$ in PMNPT and PZN-PT along nonpolar directions. Any model of origins of the strongly enhanced longitudinal response along nonpolar directions in these materials should therefore aim to explain the large shear coefficients in the crystallographic coordinate system. From the point of view of the intrinsic behavior, we see that simple perovskites and complex relaxor ferroelectrics behave qualitatively in the same way. The main difference is that the shear coefficients are anomalously large in relaxor ferroelectrics.

To gain a deeper understanding of the origins of the maximum $d_{33}^{*}(\theta, \varphi)$ along nonpolar directions, it useful to look at the thermodynamic relations for the piezoelectric coefficients derived in the framework of the LandauGinzburg-Devonshire (LGD) theory [29, 30]. More detailed discussion for all crystal phases and piezoelectric coefficients can be found elsewhere [16]. As an example, 


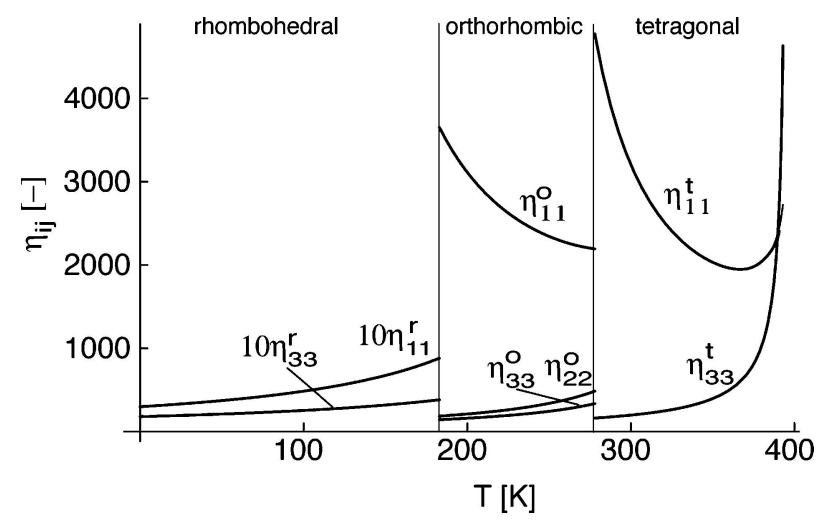

Figure 6 Predicted temperature dependence of dielectric susceptibilities in all three ferroelectric phases of $\mathrm{BaTiO}_{3}$.

only the tetragonal phase is considered here. The longitudinal and the shear coefficients can be expressed as:

$$
\begin{gathered}
d_{15}^{t}=d_{24}^{t}=\varepsilon_{0} \eta_{11}^{t} Q_{44} P_{3}^{t} \\
d_{33}^{t}=\varepsilon_{0} \eta_{33}^{t} Q_{11} P_{3}^{t}
\end{gathered}
$$

where $Q$ are the electrostrictive coefficients, $P$ is the spontaneous polarization and $\varepsilon_{0}$ the permittivity of vacuum. The temperature dependences of the susceptibilities parallel, $\eta_{33}^{t}$, and perpendicular, $\eta_{11}^{t}$, to the polarization are given in Fig. 6. The increase of the shear coefficient $d_{15}^{t}$ on the tetragonal side of the tetragonal-orthorhombic phase transition temperature is clearly caused by the anomalous behavior of the $\eta_{11}^{t}$. In analogy to pretransitional behavior in the paraelectric phase where susceptibility follows the Curie Weiss law and diverges as the cubic-tetragonal phase transition temperature is approached on cooling, the $\eta_{11}^{t}$ susceptibility in the tetragonal phase diverges as the crystal is cooled toward the tetragonal-orthorhombic phase transition temperature. Note that it is the susceptibility $\eta_{11}^{t}$ and not $\eta_{33}^{t}$ which is anomalous near the tetragonal-orthorhombic phase transition temperature, anticipating the change in the polarization direction from the [001] axis in the tetragonal phase to the pseudocubic $\langle 011\rangle$ axes in the orthorhombic state. The large value of $d_{15}^{t}$ and the large $d_{15}^{t} / d_{33}^{t}$ ratio, essential for having a maximum of $d_{33}^{t *}(\theta)$ along a nonpolar direction, then follow immediately from Equations 6, 7 and 3. Similar arguments can be invoked for the orthorhombic and rhombohedral phases [16].

The association of the large dielectric susceptibility perpendicular to the polarization with the large longitudinal response along nonpolar directions is in agreement with the results of first principle calculations. These studies interpret the enhanced piezoelectric coefficients along off-polar directions in perovskite materials by the large polarization rotation induced by the strong external electric fields [31, 32]. The above discussion shows that the polarization rotation argument is valid in the weak field limit as well: the large $\eta_{11}^{t}$ indicates enhanced polarization rotation (dielectrically soft material), and the large polarization rotation is indicative of the large shear piezoelectric coefficients [16] (see also Fig. 1). These results obtained by analyzing behavior of $\mathrm{BaTiO}_{3}$ are directly relevant to relaxor ferroelectrics, which exhibit huge shear coefficients in the crystallographic coordinate system [14, 15,22 ], and to $\mathrm{Pb}(\mathrm{Zr}, \mathrm{Ti}) \mathrm{O}_{3}$ solid solution [33] (see $\mathrm{Sec}-$ tion 4).

In this context it is interesting to consider the behavior of ferroelectric materials in the absence of any ferroelectric-ferroelectric phase transitions. In $\mathrm{PbTiO}_{3}$, which is believed to be tetragonal at all temperatures below the Curie temperature [34], the phenomenological calculations and the experimental results $[35,36]$ show that the anisotropy in $\eta_{11}^{t}$ and $\eta_{33}^{t}$ is much weaker than in the tetragonal $\mathrm{BaTiO}_{3}$ and that $d_{15}^{t}<d_{33}^{t}$ at all temperatures. The maximum $d_{33}^{*}(\theta)$ in this material appears thus always along the polar direction [16, 28]. Intriguingly, this is not the case for non-perovskite $\mathrm{LiNbO}_{3}$ that possesses rhombohedral $3 m$ symmetry below and $\overline{3} m$ above the Curie temperature. At room temperature this crystal exhibits a $d_{33}^{*}(\theta, \varphi)$ surface characteristic of rhombohedral perovskites (similar to Fig. 5) with the maximum $d_{33}^{*}(\theta, \varphi)$ approximately along the [001] pseudocubic direction and not along the polar [111] pseudocubic axis. In contrast to $\mathrm{PbTiO}_{3}$, the shear $d_{15}$ coefficient in $\mathrm{LiNbO}_{3}$ is much larger than $d_{33}$ [2]. This difference could be related to the crystal structure of $\mathrm{LiNbO}_{3}$ that can be considered as a modified perovskite structure [37], but which may be softer then the proper perovskite structure under shear fields. It would be interesting to see if there are any rhombohedral materials that exhibit the qualitative change in $d_{33}^{*}(\theta, \varphi)$ with temperature analogous to that predicted for tetragonal $\mathrm{BaTiO}_{3}$.

A further step in understanding the origins of the piezoelectric anisotropy in the framework of the phenomenological thermodynamic theory is to analyze changes of the free energy function as the crystal is cooled through the successive phase transitions. The large longitudinal piezoelectric effect along nonpolar directions can be related to the flat Gibbs free energy $G$ near the phase transition temperatures, as shown in Fig. 7. In tetragonal phase polarization is oriented along [001] axis, i.e., $P_{3} \neq 0, P_{1}$ $=P_{2}=0$ while in the orthorhombic phase $P_{3}=P_{2} \neq 0$, $P_{1}=0$. In Fig. 7 the Gibbs energy is plotted as a function of $P_{2}$ for the same temperatures as in Fig. 3, and for $P_{3}$ values corresponding to the minimum of $G$ at each temperature and giving stable tetragonal phase. The free energy becomes flatter as the tetragonal-orthorhombic phase transition is approached on cooling, indicating incipient orthorhombic phase and onset of minima in $G$ at $P_{2} \neq$ 0 at lower temperatures. The flat $G$ in the (100) plane indicates a large $1 / \eta_{i i}=\partial^{2} G / \partial P_{i}^{2}$ i.e., a large susceptibility $\eta_{11}\left(=\eta_{22}\right)$ along [100] and [010] axes (compare 


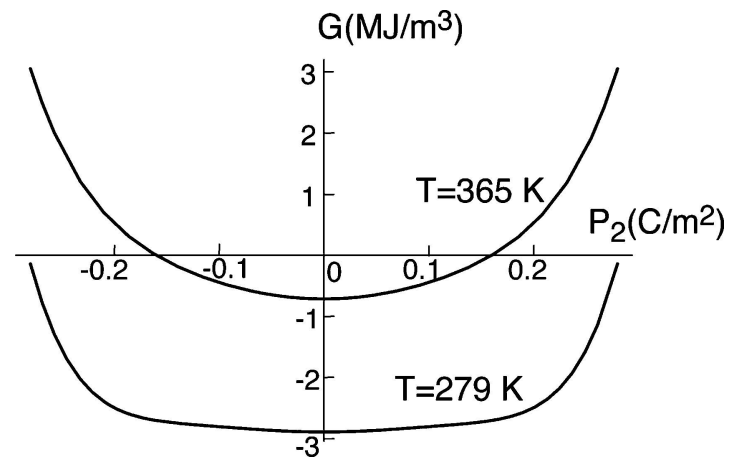

Figure 7 Gibbs free energy for tetragonal $\mathrm{BaTiO}_{3}$ at $T=365 \mathrm{~K}$ and $279 \mathrm{~K}$ (compare with Fig. 3). The energy is presented as a function of polarization $P_{2}$ along [010] axis, with polarization $P_{3}$ along [001] axis fixed at $P_{3}=0.267 \mathrm{C} / \mathrm{m}^{2}$ at $\mathrm{T}=279 \mathrm{~K}$, and fixed at $P_{3}=0.242 \mathrm{C} / \mathrm{m}^{2}$ at $T=365 \mathrm{~K}$. The minimum of $G$ at $P_{2}=0$ indicates stable tetragonal phase. The orthorhombic phase is obtained for $P_{2}=P_{3}$. The flat energy near the orthorhombic-tetragonal phase transition temperature $(T=279 \mathrm{~K})$ indicates large dielectric susceptibility $\eta_{11}^{t}$, (compare with Fig. 6) and easy polarization rotation in the $P_{2}-P_{3}$ plane.

with Fig. 6). As already discussed above, the large susceptibility perpendicular to the polar direction signifies enhanced polarization rotation and large shear piezoelectric coefficients (compare with Figs 2 and 6). A similar approach can be also applied for the free energy evolution as a function of the composition in solid solutions [38-42], and as a function of external fields [27]. Another approach is to use first principle calculations, and to attempt to explain the dielectric, elastic and piezoelectric properties of the perovskite structure in terms of ionic and electronic contributions to polarization [31, 43-47].

\section{Piezoelectric anisotropy-composition relationships in materials exhibiting a morphotropic phase boundary}

The previous section discussed enhancement of the piezoelectric anisotropy as a function of the proximity of the phase transition temperatures. In analogy, one can expect to observe similar effects in the vicinity of the compositionally driven phase changes in materials exhibiting a morphotropic phase boundary (MPB) [42]. Fig. 8 shows the evolution of $d_{33}^{t}, d_{31}^{t}$, and $d_{15}^{t}$ piezoelectric coefficients and $\eta_{11}^{t}$ and $\eta_{33}^{t}$ dielectric susceptibilities in single crystals of $\mathrm{Pb}(\mathrm{Zr}, \mathrm{Ti}) \mathrm{O}_{3}$ (PZT) solid solution, as a function of composition, on the tetragonal side of the MPB. In this diagram, which is derived using the LGD thermodynamic phenomenological theory, the MPB was chosen to appear at a 50:50 $\mathrm{Ti} / \mathrm{Zr}$ ratio [48]. At compositions close to the $\mathrm{MPB}$, where polarization changes direction from the [001] axis (tetragonal side) to the [111] quasicubic direction (rhombohedral side), $d_{15}^{t}$ and $\eta_{11}^{t}$ strongly increase, just as in tetragonal $\mathrm{BaTiO}_{3}$ near the tetragonal-orthorhombic phase transition temperature (compare Fig. 8 with Figs 2 and 6). Note that coefficients of the LGD function for PZT

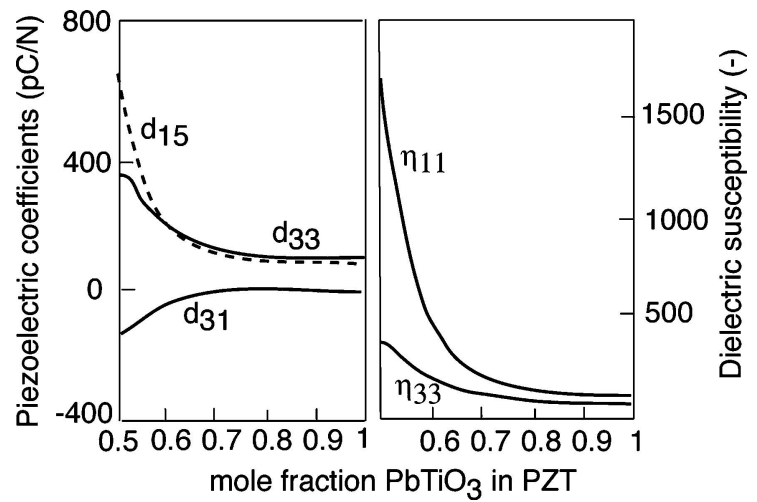

Figure 8 Piezoelectric coefficients and dielectric susceptibilities in $\mathrm{Pb}(\mathrm{Zr}, \mathrm{Ti}) \mathrm{O}_{3}$ crystals calculated using the LGD theory as a function of composition, for the tetragonal side of the morphotropic phase boundary. After [48].

are determined experimentally, that is, they are independent on the recent discovery of the monoclinic distortion in PZT, except in the region very close to the MPB where rhombohedral and tetragonal symmetries are assumed. Values of coefficients will change if measured more precisely and if presence of the monoclinic phase was taken into account, however, trends indicated in Figs 7 to 9 should qualitatively remain the same, especially for compositions not lying too close to the MPB.

While in the case of tetragonal PZT the $d_{15}^{t}$ fails to reach a high enough value [28] to bring the maximum $d_{33}^{t *}(\theta)$ away from the [001] direction, the general tendency is similar to that in the tetragonal $\mathrm{BaTiO}_{3}$, as illustrated in Fig. 9: as the $\mathrm{Zr}$ content increases and the crystal approaches the MPB, the $d_{33}^{t *}(\theta)$ becomes flatter at the top, indicating tendency toward developing a local minimum along the [001] axis and a maximum away from this direction. As mentioned above, it is very likely that the coefficients of the Gibbs energy are not optimized in the case of PZT and for this reason the maximum in the calculated $d_{33}^{t *}(\theta)$ is not obtained along a nonpolar direction at compositions very close to the MPB.

A similar tendency is calculated for the rhombohedral side of the MPB in PZT. Fig. 10 shows the $d_{33}^{r *}(\theta, \varphi)$ surface and its cross section for two rhombohedal compositions with $\mathrm{Zr} / \mathrm{Ti}$ ratio $90 / 10$ and 60/40, at room temperature. The composition 90/10, which lies well into the rhombohedral side of the phase diagram, demonstrates a reduced piezoelectric anisotropy with respect to the composition 60/40 that is close to the MPB. Unfortunately, PZT crystals are not available and these theoretical predictions cannot be verified experimentally. However, reports on the piezoelectric properties of highly textured thin films do show a maximum in $d_{33}^{r *}(\theta, \varphi)$ approximately along the quasicubic [001] direction in 60/40 PZT composition and not along the polar quasicubic [111] axis, as shown in Fig. 11 [49]. Note that the former orientation also exhibits weaker nonlinearity with respect to the 

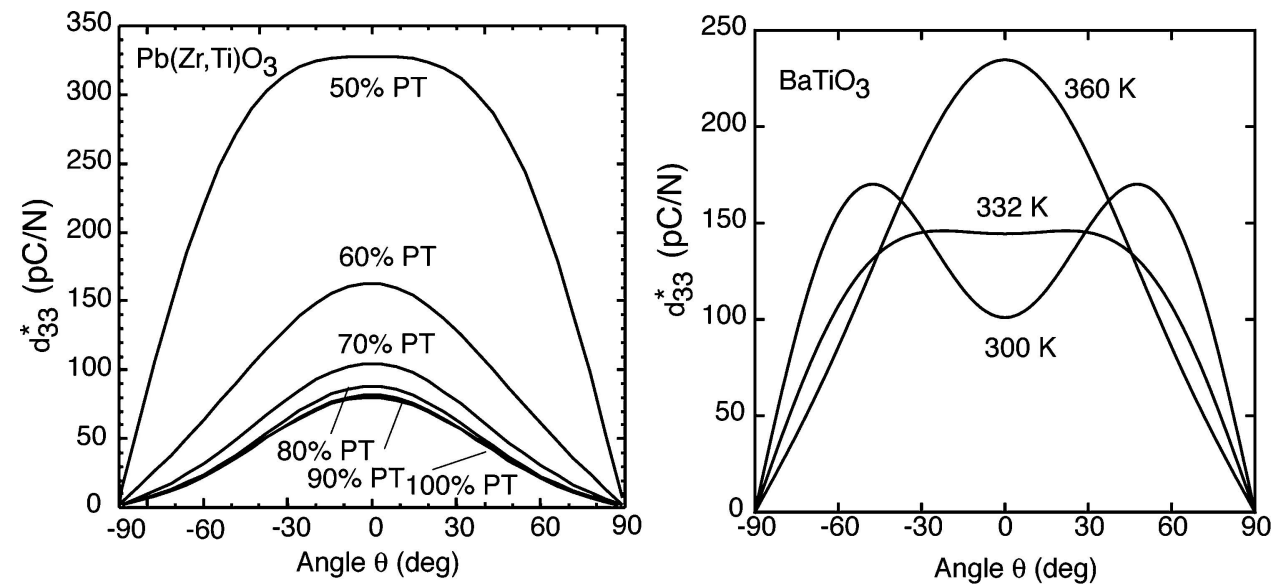

Figure 9 On the left: orientation dependence of $d_{33}^{t *}(\theta)$ for $\mathrm{Pb}(\mathrm{Zr}, \mathrm{Ti}) \mathrm{O}_{3}$ ( $\left.\mathrm{PZT}\right)$ at room temperature as a function of $\mathrm{PbTiO}_{3}(\mathrm{PT})$ concentration, on the tetragonal side of the MPB. On the right: orientation dependence of $d_{33}^{t *}(\theta)$ for tetragonal $\mathrm{BaTiO}_{3}$ at different temperatures. Note similarities between $d_{33}^{t *}(\theta)$ at $60 \% \mathrm{PT}$ in PZT and at $360 \mathrm{~K}$ in $\mathrm{BaTiO}_{3}$; and at $50 \% \mathrm{PT}$ in PZT and at $332 \mathrm{~K}$ in $\mathrm{BaTiO}_{3}$. Data in both figures were calculated for mono domain single crystals using the LGD approach. LGD parameters for PZT were taken from Ref. [48].
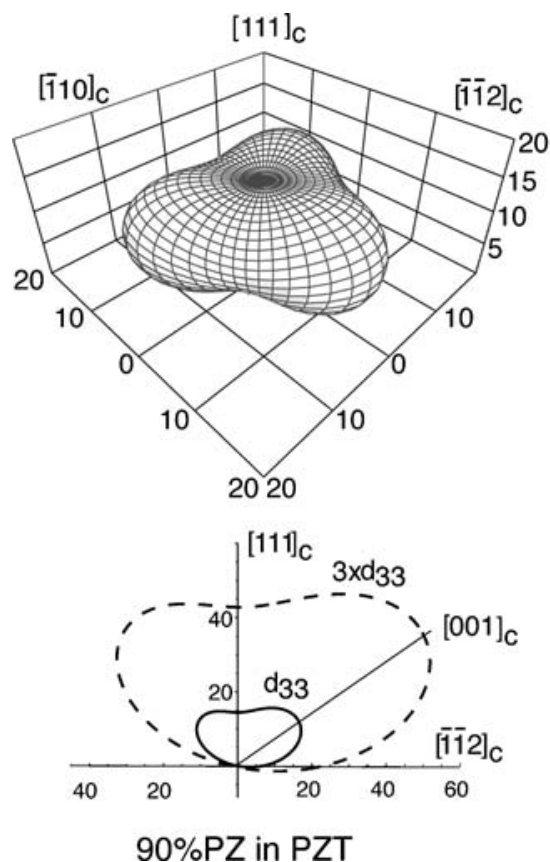
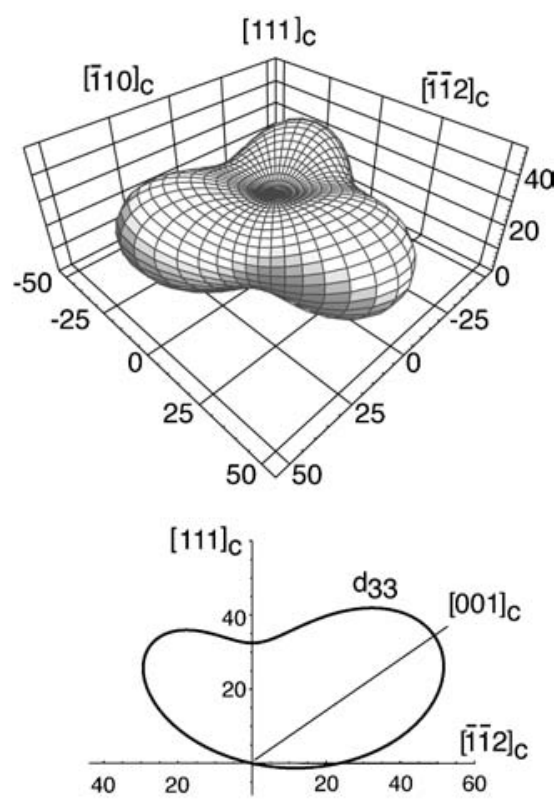

$60 \% \mathrm{PZ}$ in PZT

Figure 10 Orientation dependence of $d_{33}^{t *}(\theta, \varphi)$ for two rhombohedral compositions of $\mathrm{PZT}$, one containing $90 \% \mathrm{PbZrO} 3(\mathrm{PZ})$ and the other $60 \% \mathrm{PZ}$. Cross section of the surfaces is shown in the bottom part of the figure. Note the reduced anisotropy in the composition with $90 \%$ PZ, which lies deep into rhombohedral part of the phase diagram. Dashed curve for this composition shows data magnified by factor three, for better comparison with the composition containing 60\% PZ. Data were calculated at room temperature, for mono domain single crystals using the LGD approach. The LGD parameters were taken from Ref. [48].

driving field amplitude, which is consistent with the small contribution of domain walls to the piezoelectric effect. (Sections 6 and 7).

\section{Effect of external electric fields on piezoelectric anisotropy}

The effect of an electric field on the piezoelectric properties of perovskite crystals has been recently examined in $\mathrm{BaTiO}_{3}$ by first principle calculations [31] and LGD the- ory [27], and in PZT by first principle calculations [32]. Results show that the application of the field along nonpolar directions enhances $d_{33}^{*}(\theta, \varphi)$ and other piezoelectric coefficients. In the case of very large fields, the field induced phase transitions are accompanied by huge shear piezoelectric coefficients, and thus a large $d_{33}^{*}(\theta, \varphi)$ along nonpolar directions [32].

These results and the discussion in previous sections suggest that the enhanced $d_{33}^{*}(\theta, \varphi)$ along nonpolar direc- 


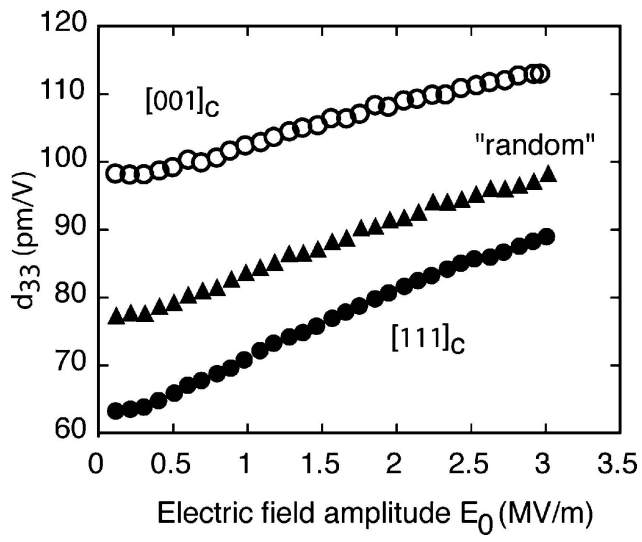

Figure 11 The longitudinal piezoelectric coefficient in textured 60PZ/40PT thin films as a function of the driving electric field amplitude. The largest response is observed in films with $[001]_{\mathrm{c}}$ preferential orientation, in qualitative agreement with results predicted for single crystals. The relatively smaller piezoelectric nonlinearity of the sample with $[001]_{\mathrm{c}}$ orientation is consistent with reduced contribution of domain walls for this orientation. For details see Ref. [49].

tions may be associated with instabilities related to incipient phase transitions, which are manifested by dielectric softening in directions perpendicular to the polarization axis. In this section we show that such instabilities may be also induced by application of the electric field bias applied antiparallel to polarization, leading to a huge enhancement of the $d_{33}^{*}(\theta, \varphi)$.

For simplicity, we again consider the tetragonal phase of a mono domain $\mathrm{BaTiO}_{3}$ single crystal under electric bias field applied either along the polar axis [001] or antiparallel to it, i.e. along the [001] direction. The effect of the field on the piezoelectric coefficients and susceptibilities is shown in Fig. 12. Calculation details can be found in Ref. [50]. The orientation dependence of $d_{33}^{t *}(\vartheta)$ can be calculated using Equation 3 and is shown in Fig. 13 for different bias fields at $T=285 \mathrm{~K}$ and $365 \mathrm{~K}$. The $d_{33}^{*}(\vartheta)$ exhibits its maximum value for all bias fields at approximately $\vartheta_{\max } \approx 50^{\circ}$, i.e. close to the [111] axis. The value of $d_{33}^{t *}\left(\vartheta_{\max }\right)$ depends strongly on the bias field. For $E_{3}$ $=0, d_{33}^{t *}\left(\vartheta_{\max }\right)=227 \mathrm{pm} / \mathrm{V}$. While positive bias fields decrease $d_{33}^{t *}\left(\vartheta_{\max }\right)$, the calculations predict that negative bias fields (anti-parallel to polarization) strongly enhance the piezoelectric coefficient. A field of $-9 \mathrm{MV} / \mathrm{m}$ (applied along [001]) increases $d_{33}^{*}\left(\vartheta_{\max }\right)$ to $497 \mathrm{pm} / \mathrm{V}$, which represents more than a five fold increases with respect to the value measured along the polar axis at zero bias field [ $\left.d_{33}^{*}\left(\vartheta=0^{\circ}\right)=d_{33}=89 \mathrm{pm} / \mathrm{V}\right]$ and more than a two fold increase with respect to the maximum value measured at $E_{3}=0\left[d_{33}^{*}\left(\vartheta=50^{\circ}\right)=227 \mathrm{pm} / \mathrm{V}\right]$.

The above results can be again understood by analyzing Equations 6-7 and the field dependences of the susceptibilities and piezoelectric coefficients, shown in Fig. 12. The negative bias fields strongly increase $\eta_{11}$ and, consequently, lead to a high $d_{15}$ coefficient, which is then directly responsible for a maximum of $d_{33}^{*}(\vartheta)$

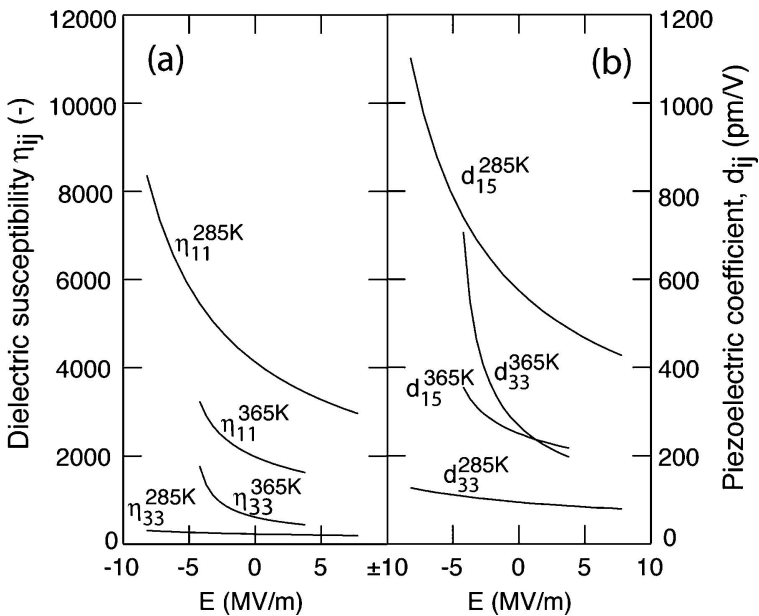

Figure 12 (a) Dielectric suscpetibilities and (b)piezoelectric coefficients of the tetragonal $\mathrm{BaTiO}_{3}$ at two temperatures as a function of the electric field bias. The negative field is applied against the spontaneous polarization. The field dependence was calculated using the LGD theory. For details, see Ref. [50].

along a nonpolar direction. In contrast to earlier studies where strong bias fields were always applied along nonpolar directions, these results show that a weak-field polarization rotation may be facilitated by application of strong bias fields anti-parallel to polarization. It has been shown in Section 3 that the proximity of the tetragonalorthorhombic phase transition temperature by itself leads to a maximum $d_{33}^{t *}\left(\vartheta_{\max }\right)$ approximately along the [111] axis, however, we now show that this effect is enhanced considerably by anti-parallel bias fields.

Interesting results are also observed at higher temperatures, closer to the tetragonal-cubic phase transition that occurs at $393 \mathrm{~K}$. At these temperatures the condition for having a maximum in $d_{33}^{t *}\left(\vartheta_{\max }\right)$ along a non-polar direction is not fulfilled [28] and the piezoelectric response exhibits its maximum value along the polar axis. The positive bias field decreases the piezoelectric coefficient, similarly to what has been reported in ferroelectric thin films [51], while the negative field enhances the piezoelectric response, Fig. 13. At $E_{3}$ $=-4 \mathrm{MV} / \mathrm{m}$ and at $365 \mathrm{~K}$, the maximum $d_{33}^{*}(\vartheta=0)$ is $700 \mathrm{pm} / \mathrm{V}$, compared to $268 \mathrm{pm} / \mathrm{V}$ at $E_{3}=0$. Note that this field enhancement of the piezoelectric coefficient is not related to the polarization rotation.

It is interesting to note the similarities among the temperature, composition and field dependences of the piezoelectric coefficients in Figs 2, 8 and 12. The common denominator for each case is the increase of the susceptbility i.e., the dielectric softening of the crystal, perpendicular to the polarization. This in turn leads to an increase in the shear piezoelectric coefficients and enhanced $d_{33}^{t *}(\vartheta)$ along nonpolar directions. As discussed above, the dielectric softening is the direct consequence of the flattening of the Gibbs free energy. 

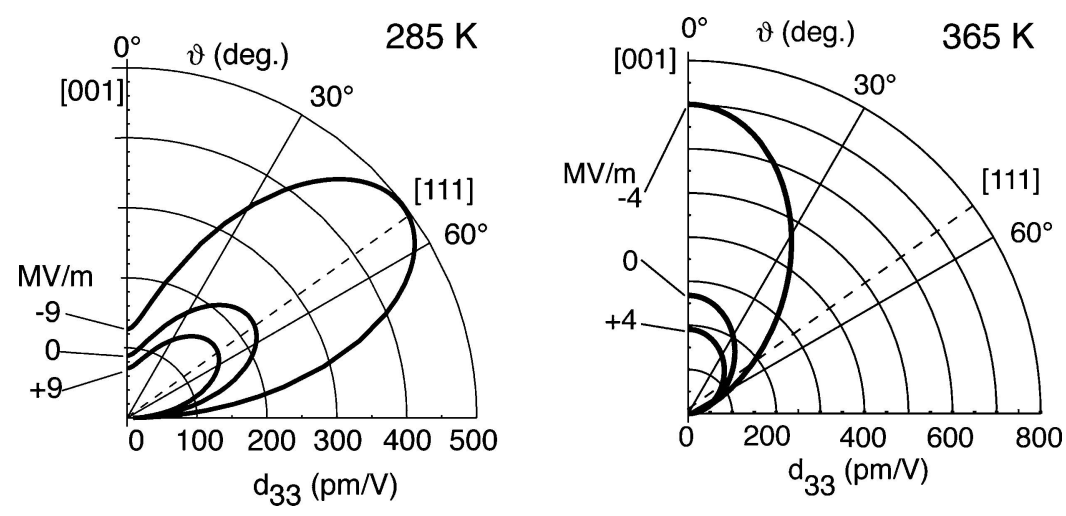

Figure 13 Orientation dependence of $d_{33}^{t *}(\theta)$ for tetragonal $\mathrm{BaTiO}_{3}$ at two temperatures and for different electric fields applied along polarization (positive fields) and antiparellel to polarization (negative fields). The field dependence was calculated using the LGD theory. For details, see Ref. [50].

Finally, one obtains similar effects under applied external stresses [52]. For example, calculations on thin films show a large increase in the shear piezoelectric coefficients due to misfit strains [53] which reflects on the orientation dependence of piezoelectric coefficients.

\section{Effect of domain wall structure on piezoelectric anisotropy}

In previous sections we have discussed the orientation dependence of piezoelectric properties assuming that the crystal is in the mono domain state. Practically, the mono domain state is difficult to achieve in obliquely cut crystals, and in relaxor ferroelectrics the monodoman state appears to be unstable [14]. When a crystal is cut and poled along special directions so that the resulting domain states are equivalent and the piezoelectric properties are enhanced, the resulting domain structure is often called the "engineered domain state" [7]. Clearly there can be more than one "engineered domain state" for a given symmetry. In tetragonal crystals with polarization along the [001] axis, one of these states is obtained by poling the crystal along one of the $\langle 111\rangle$ axes. In rhombohedral materials with polarization along the $\langle 111\rangle$ quasicubic directions, an engineered domain state can be obtained by poling the crystal along the [001] quasicubic axis. In this case, four equivalent domain states are defined by polarization vectors oriented along the four equivalent

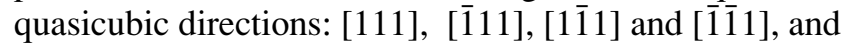
are shown in Fig. 14. With respect to an electric field applied along the poling direction, i.e. along the [001] quasicubic axis, these rhombohedral domain states are energetically equivalent and the associated domain walls are expected to be stable. Likewise, this rhombohedral domain-engineered state should be stable to stress: uniaxial pressure applied along the [001] psuedocubic direction will not favor any one of the four domain states.

The question is posed whether the high properties in poled relaxor ferroelectric crystals are a simple conse-

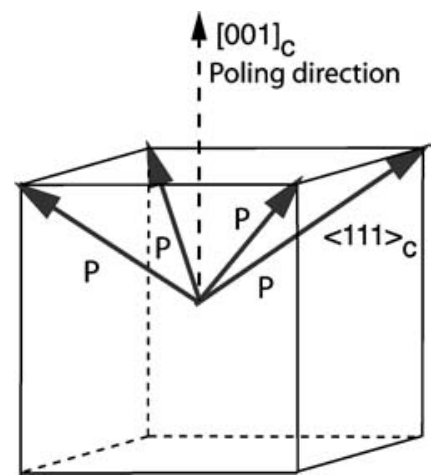

Figure 14 Schematic representation of the equivalent polarization directions in a rhombohedral $3 m$ crystal poled along quasicubic $[001]_{\mathrm{c}}$ direction. For more examples and discussion see Ref. [54].

quence of the material anisotropy, or whether the "engineered domain state" somehow further enhances the properties along nonpolar directions beyond what is expected from the intrinsic anisotropy. Considering the equivalence of the engineered domain states, the associated domain walls should be immobile and should not contribute to the piezoelectric properties. An indication that this is indeed so is found in the anhysteretic converse piezoelectric response of relaxor ferroelectric single crystals with engineered domain states (see [7] and Section 7) and in the reduced nonlinearity of piezoelectric properties in highly textured thin films, Fig. 11 [49].

This question was first addressed by Nakamura et al. $[11,55]$. They showed that in tetragonal $\mathrm{BaTiO}_{3}$ and orthorhombic $\mathrm{KNbO}_{3}$ crystals the piezoelectric response in crystals with an engineered domain state is approximately the same as in mono domain crystals cut and measured along the same direction. Later, the same was shown [15, 22] for $0.67 \mathrm{~Pb}\left(\mathrm{Mg}_{1 / 3} \mathrm{Nb}_{2 / 3}\right) \mathrm{O}_{3}-0.33 \mathrm{PbTiO}_{3}$ crystals although this case is perhaps more controversial. Firstly, as a function of preparation and poling conditions, this latter composition can exhibit several different crystal structures [56] and secondly, in order to stabilize the mono 


\section{FRONTIERS OF FERROELECTRICITY}

domain state, the properties of the crystal had to be measured under an electric bias field [15]. It is interesting to note that in all cases mentioned above the response of monodomain samples along the nonpolar directions was calculated and not measured.

Only recently, Wada et al. [54, 57] have carried out a systematic study of effects of engineered domain structure on properties of perovskite crystals. They showed that in $\mathrm{BaTiO}_{3}$ and $\mathrm{KNbO}_{3}$ crystals the transverse piezoelectric response of crystals with an engineered domain structure depends on the density of domain walls, while the domain structure remains the same. The piezoelectric $d_{31}$ coefficient of crystals with a coarse domain structure are similar to those of monodomain crystals measured along the same crystallographic direction. However, as the domain structure becomes finer, the response of the multidoman crystal becomes significantly higher than that of the monodomain sample. Considering the supposed equivalence of the domain states in crystals with engineered domain states this result, while significant, is unexpected and puzzling.

It is interesting to speculate about possible origins of the enhancement of piezoelectric response in crystals with dense, engineered domain walls if the contribution of domain wall displacement is, as usually assumed, excluded. One possibility is that as the number of domain walls increases, the material within the domains is no longer the same, i.e. its properties are different to those of the mono domain crystal or coarse-structured material. This can happen because with a denser domain wall structure the compensation of charges or stresses at the domain walls [58] may require more material to be involved in the compensation process; alternatively, internal fields associated with the compensation process may be stronger in crystal with finer domains. The previous sections have shown that antiparallel electric fields can enhance the intrinsic anisotropy. Additionally, the domain walls present, in a sense, a phase transition between two states; again, the transition related instabilities, discussed in previous sections, may lead to enhanced dielectric and piezoelectric properties.

\section{Piezoelectric anisotropy and domain wall displacement}

One of the most interesting features of crystals with "engineered domain states" is the absence of hysteresis in the piezoelectric strain-electric field relation, as illustrated in Fig. 15 for a rhombohedral crystal with nominal composition 0.67PMN-0.33PT. In ferroelectric materials the strain-electric field hysteresis is usually associated with the movement of domain walls. As mentioned in the previous section, in tetragonal and rhombohedral crystals with the special engineered domain structure the domain walls are not expected to move and their response should therefore be anhysteretic, as shown in Fig. 15.

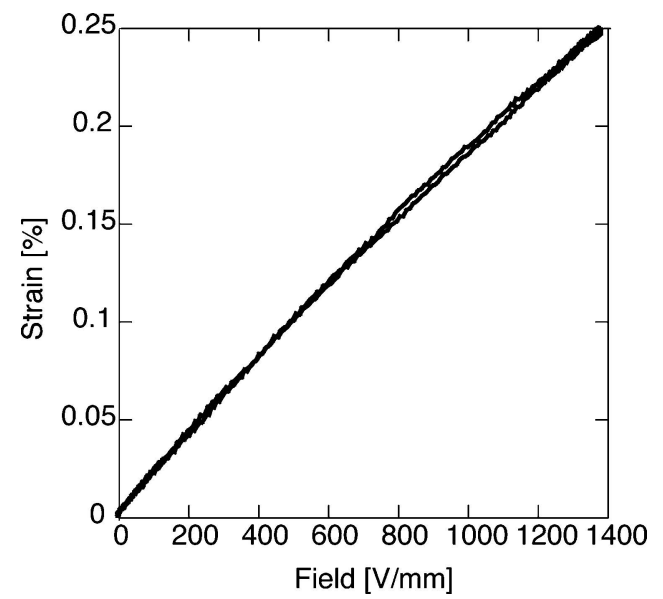

Figure 15 Anhysteretic strain-electric field relation in a 0.67PMN-0.33PT crystal poled and measured with unipolar field applied along the [001] pseudocubic direction.

It has, however, been reported that the strain-electric field relationship becomes hysteretic under uniaxial compressive stress [59], indicating a possible contribution from the moving domain walls. This fact does put into doubt the widely accepted picture, Fig. 14, of the engineered domain structure with equivalent sets of domain walls whose motion will not lead to sample size change. Note that, in the first approximation, the associated domain structure should be insensitive to both uniaxial pressure and electric field applied along the [001] quasicubic direction.

An investigation of the direct piezoelectric effect [60] has shown that in several compositions of PZN-PT and PMN-PT crystals poled along the [001] quasicubic orientation the longitudinal piezoelectric charge-force response is hysteretic and nonlinear, Fig. 16. Pressed crystals will even (partially) depole at sufficient uniaxial stresses. These experimental results may again be incompatible with the simple, usually assumed picture of the engineered domain structure. One can speculate that the absence of hysteresis in the converse effect may not necessarily be due to the presence of energetically equivalent engineered domain states, but rather due to pinned domain walls, which can be released by uniaxial compressive field, but not by unipolar electric field applied along the poling direction. If this is true then the simple picture of equivalent domain states no longer holds, and the actual domain wall structure in crystals with engineered domain states is more complex than is usually assumed.

Interestingly, in some cases (e.g., 0.68PMN-0.32PT and $0.045 \mathrm{PZN}-0.955 \mathrm{PT}$ ) it was observed that the direct transverse piezoelectric response could be anhysteretic even when the longitudinal response is hysteretic, Fig. 17. The difference between the two cases is that in the transverse response the compressive static and dynamic pressures are applied perpendicular to the poling direction, whereas in 

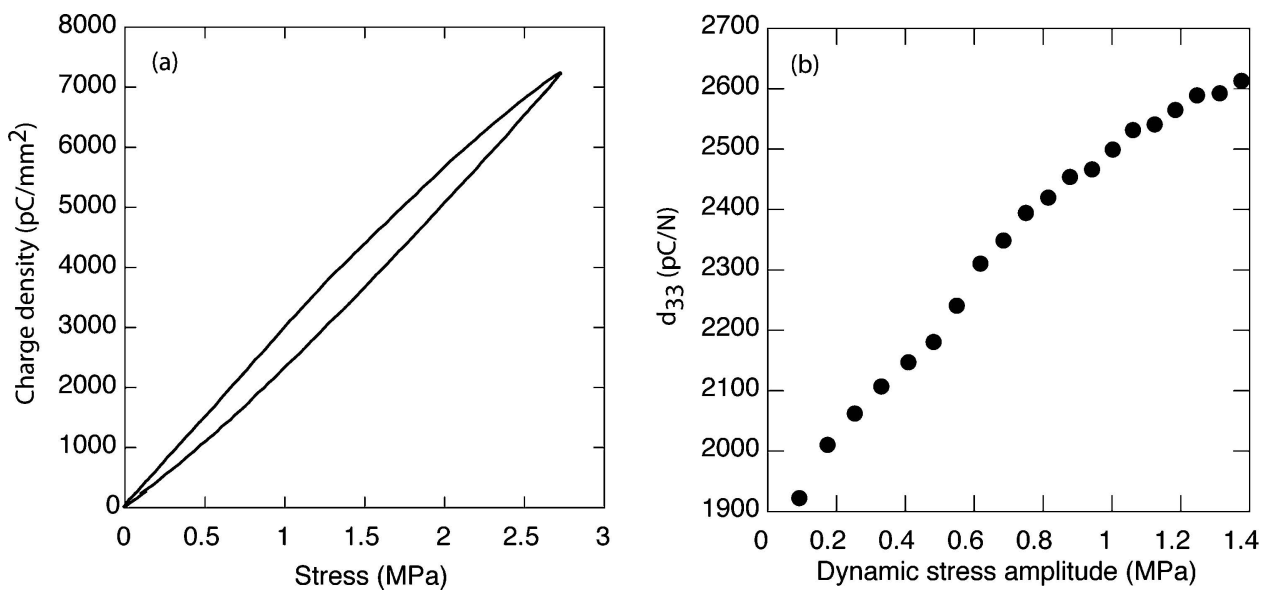

Figure 16 (a) Hysteretic charge density-stress relation and (b) field dependence of the piezoelectric coefficient in 0.67PMN-0.33PT single crystal poled and measured along the [001] quasicubic axis. For details see Ref. [60].
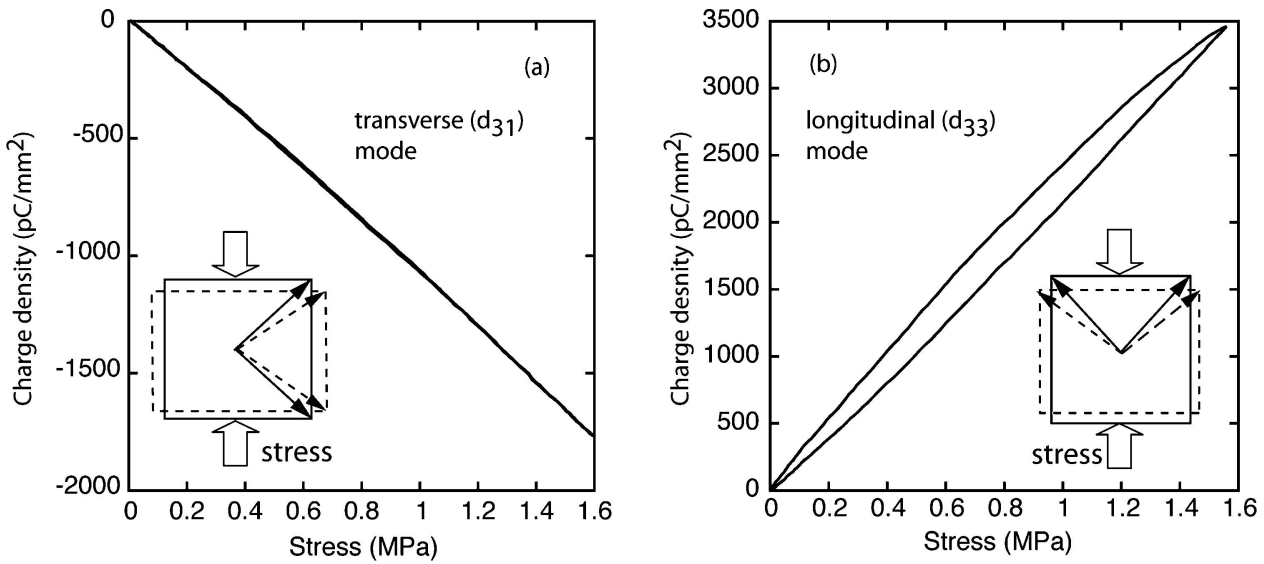

Figure 17 (a) Anhysteretic charge density-stress response for the transverse $\left(d_{31}\right)$ mode, and (b) hysteretic charge-stress response for the longitudimal $\left(d_{33}\right)$ mode in 0.68PMN-0.32PT crystals poled along [001] quasicubic axis. The diagram within each figure shows schematically direction of applied dynamic stress and presumed polarization rotation. Irrespective of the applied compressive stress, the slope of the charge-stress response is chosen to illustrate the positive sign of the $d_{33}$ and negative sign of the $d_{31}$ coefficient. For details, see Ref. [60].

the longitudinal response the pressure is applied along the quasicubic [001] poling axis. Such stresses in the transverse response will rotate the polarization vectors toward the poling direction, therefore having the same action as an electric field applied along the [001] axis. In the longitudinal case, however, compressive stresses will rotate polarization vectors away from the poling direction (see diagrams in Fig. 17). In either case the rotation direction, or indeed the rotation path, appears to be important. This apparent anisotropy in the extrinsic piezoelectric behavior should be verified for other perovskite materials.

\section{Conclusions}

Intrinsic piezoelectric anisotropy, manifest in the strong orientation dependence of the piezoelectric coefficients, is present in many simple and complex perovskites. The anisotropy can be associated to instabilities in crystals, either near temperature induced phase transitions, phase changes at the morphotropic phase boundary, or under external fields. In crystals with engineered domain states, the anisotropy can be dominated by the intrinsic anisotropy of the monodomain crystals if the domain structure is coarse. However, it appears that the anisotropy can be further enhanced when the poled crystal possesses a fine engineered domain structure.

The usual picture of the simple domain structure in crystals with engineered domain state and immobile domain walls appears to be questionable. Under external compressive pressure both the longitudinal converse and the direct piezoelectric effect become hysteretic suggesting that the domain walls are mobile. In contrast, the stress-free converse piezoelectric effect with a unipolar electric field applied along the poling axis, and, at least in some cases, the direct piezoelectric transverse effect where compressive pressure is applied in a direction perpendicular to the poling axis, are anhysteretic. 


\section{References}

1. J. C. BRICE, Rev. Mod. Phys. 57 (1985) 105.

2. T. IKEDA, "Fundamentals of piezoelectricity" (Oxford Unversity Press, Oxford, 1990)

3. H. TAKEUCHI, S. JYOMURA, E. YAMAMOTO and Y. ITO, J. Acoust. Soc. Am. 72 (1982) 1114

4. D. DAMJANOVic, T. R. GURURAJA and L. E. CROSS, Am. Ceram. Soc. Bull. 66 (1987) 699.

5. T. TAKENAKA, K. SAKATA and K. TODA, Jpn.J.Appl. Phys. 24 (1985) 730.

6. R. NEWNHAM, D. P. SKINNER and L. E. CROSS, Mat. Res. Bull. 13 (1978) 52.

7. S. E. PARK and T. R. SHROUT, J. Appl. Phys. 82 (1997) 1804

8. J. KUWATA, K. UCHINO and S. NOMURA, Jpn. J. Appl. Phys. 21 (1982) 1298.

9. M. KAHN, R. P. INGEL and D. LEWIS III, Ferroelectrics 102 (1990) 225.

10. S. WADA, S. SUZUKI, T. NOMA, T. SUZUKI, M. OSADA, M. KAKIHANA, S.-E. PARK, L. E. CROSS and T . R. SHROUT, Jpn. J. Appl. Phys. 38 (1999) 5505.

11. K. NAKAMURA, T. TOKIWA and Y. KAWAMURA, J. Appl. Phys. 91 (2002) 9272.

12. S. ZHANG, C. A. RANDALL and T. R. SHROUT, ibid. 95 (2004) 4291.

13. S. ZHANG, P. W. REHRIG, C. A. RANDALL and T. R. SHROUT, J. Crystal Growth 234 (2002) 415.

14. R. ZHANG, B. JIANG and W. CAO, Appl. Phys. Lett. 82 (2003) 787.

15. Idem. ibid. 82 (2003) 3737.

16. M. BUDIMIR, D. DAMJANOVIC and N. SETTER, $J$. Appl.Phys. 74 (2003) 6753.

17. B. NOHEDA, D. E. COX, G. SHIRANE, J. A. GONZALO, L. E. CROSS and S. -E. PAR K, Appl. Phys. Lett. 74 (1999) 2059.

18. E. M. SABOLSKY, A. R. JAMES, S. KWON, S. TROLIER-MCKINSTRY and G. L. MESSING, ibid. 78 (2001) 2553.

19. J. F. NYE, "Physical Properties of Crystals," (Oxford University, Oxford, 1985).

20. H. GOLDSTEIN, "Classical Mechanics," (Addison-Wesley, Reading, MA, 1978).

21. D. DAMJANOVIC, M. BUdIMIR, M. DAVIS and N. SETTE R, Appl. Phys. Lett. 83 (2003) 2490.

22. Idem. ibid. 83 (2003) 527.

23. S. ZHANG, L. LAURENT, S. LIU, S. RHEE, C. A. RANDALL and T. R. SHROUT, Jpn. J. Appl. Phys. 41 (2002) L1099.

24. Y. I. SIROTIN and M. P. SHASKOLSKAYA, "Fundamentals of crystal physics," (Mir, Moscow, 1982).

25. M. E. LINES and A. M. GLASS, "Principles and Applications of Ferroelectrics and Related Materials," (Clarendon, Oxford, 1979).

26. A. GARCIA and D. VANDER BILT, Appl. Phys. Lett. 72 (1998) 2981.

27. A. J. BELL, J. Appl. Phys. 89 (2001) 3907.

28. D. DAMJANOVIC, F. BREM and N. SeTter, Appl. Phys. Lett. 80 (2002) 652

29. A. F. DEVONShire, Phil. Mag. 40 (1949) 1040.

30. Idem., ibid. 42 (1951) 1065.

31. H. FU and R. E. COHEN, Nature $\mathbf{4 0 3}$ (2000) 281.

32. L. Bellaiche, A. GARCiA and D. VAnderbilt, Phys. Rev. B 64 (2001) 060103.
33. R. GUO, L. E. CROSS, S.-E. PARK, B. NOHEDA, D. E. COX and G. SHIRANE, Phys. Rev. Lett. 84 (2000) 5423.

34. J. Kobayashi, Y. UeSU and Y. SAKemi, Phys. Rev. B 28 (1983) 3866.

35. M. J. HAUN, E. FURMAN, S. J. JANG, H. A. MCKINSTRY and L. E. CROSS, J. Appl. Phys. 62 (1987) 3331.

36. Z. Li, M. GRIMSDITCh, X. XU and S.-K. CHAN, Ferroelectrics 141 (1993) 313.

37. J. M. HER B ERT, "Ferroelectric Transducers and Sensors," (Gordon and Breach, New York, 1982) Vol. 3.

38. Y. ISHIB A S HI and M. IWATA, Jpn. J. Appl. Phys. 37 (1998) L985.

39. Y. ISHIBASHI and M. IWATA, Japanese Journal of Applied Physics Part 1-Regular Papers Short Notes \& Review Papers 38 (1999) 800.

40. M. IWATA and Y. IS HIB A S HI, Jpn. J. Appl. Phys. Part 1 38(9B), (1999) 5670.

41. M. IWATA and Y. ISHIBASHI, Japanese Journal of Applied Physics Part 1-Regular Papers Short Notes \& Review Papers 39 (2000) 5156.

42. M. IWATA, H. ORIHARA and Y. ISHIBASHI, Ferroelectrics 266 (2002) 57.

43. D. VANDERBILT, ibid. 301 (2004) 9.

44. L. Bellaiche, A. GARCiA and D. VAnderillt, Phys. Rev. Lett. 84 (2000) 5427.

45. L. BeLlaiche and D. VANDERBILT, ibid. 83 (1999) 1347.

46. G. S AGHI-SZAB O and R. E. COHEN, Ferroelectrics 194 (1997) 287.

47. R. E. COHEN, Nature 358 (1992) 136

48. M. J. HAUN, E. FURMAN, S. J. JANG and L. E. CROSS, Ferroelectrics 99 (1989) 63.

49. D. V. TAYLOR and D. DAMJANOVIC,Appl. Phys. Lett. 76 (2000) 1615 .

50. M. BUdimir, D. DAMJANOVIC and N. SETter, ibid. 85 (2004) 2890.

51. L. CHEN, V. NAGARAJAN, R. RAMESH and A. L. ROYTB U R D, J. Appl. Phys. 94 (2003) 5147.

52. M. BUDIMIR, D. DAMJANOVIC and N. SETTER, (2005) Unpublished.

53. V. G. KOUKHAR, N. A. PERTSEV and R. WASER, Phys. Rev. B 64 (2001) 214103.

54. S. WADA, H. KAKEMOTO and T. TSURUMi, Mater. Trans. 45 (2004) 178.

55. K. NAKAMURA, T. TOKIWA and Y. KAWAMURA, in "Proceedings of the 12th IEEE International Symposium on the Applications of Ferroelectrics," (IEEE Service Center, Honolulu, 2000) Vol. II, pp. 717.

56. Y. LU, D.-Y. JEONG, Z.-Y. CHENG, Q. M. ZHANG, H.S. LUO, Z.-W. YIN and D. VIEHLAND, Appl. Phys. Lett. 78 (2001) 3109

57. S. WADA, K. MURAOKA, H. KAKEMOTO, T. TSURUMI and H. KUMAGAI, Jpn. J. Appl. Phys. $\mathbf{4 3}$ (2004) in print.

58. V. Y. TOPOLOV, J. Phys.-Cond. Matter 16 (2004) 2455.

59. D. VIEHLAND, L. EWART, J. POWERS and J. F. Li, $J$. Appl. Phys. 90 (2001) 2479.

60. M. DAVis, D. DAMJANOVIC and N. SETTER, J. Appl. Phys. 95 (2004) 5679 